PROCEEDINGS OF THE

AMERICAN MATHEMATICAL SOCIETY

Volume 139, Number 11, November 2011, Pages 3915-3927

S 0002-9939(2011)10855-4

Article electronically published on March 10, 2011

\title{
HOLOMORPHIC AUTOMORPHISMS OF DANIELEWSKI SURFACES I - DENSITY OF THE GROUP OF OVERSHEARS
}

\author{
FRANK KUTZSCHEBAUCH AND ANDREAS LIND
}

(Communicated by Franc Forstnerič)

\begin{abstract}
We define the notion of shears and overshears on a Danielewski surface. We show that the group generated by shears and overshears is dense (in the compact open topology) in the path-connected component of the identity of the holomorphic automorphism group.
\end{abstract}

\section{INTRODUCTION}

In the early 90's, Andersén and Lempert started intensive studies of the automorphisms group $\mathrm{Aut}_{\mathrm{hol}}\left(\mathbb{C}^{n}\right)$ of $\mathbb{C}^{n}, 3$ and 4 . They showed (thus answering a question by Rosay and Rudin [18]) that the subgroup generated by shears and overshears $\operatorname{OS}\left(\mathbb{C}^{n}\right)$ is dense in the holomorphic automorphism group $\mathrm{Aut}_{\text {hol }}\left(\mathbb{C}^{n}\right)$, although it is a proper subgroup of $\operatorname{Aut}_{h o l}\left(\mathbb{C}^{n}\right)$ in all dimensions $n \geq 2$. A special feature in dimension 2 is that the group $\operatorname{OS}\left(\mathbb{C}^{2}\right)$ possesses the structure of a free amalgamated product of the affine automorphisms and of the Jonquiere automorphisms (proved by Ahern and Rudin [2]). This structure theorem is analogous to the classical structure theorem for the polynomial automorphism group of $\mathbb{C}^{2}$ due to van der Kulk 19 and Jung [10].

In recent years Varolin, 20, extended the notion of shears and generalized the Andersén-Lempert theory for automorphisms of $\mathbb{C}^{n}$ to a larger class of Stein manifolds, namely manifolds with the density property; see [21] and 22. Continuing Varolin's research, the first author and Kaliman ([11 and [12]) showed that this class of manifolds is quite large and that it is an important source of examples concerning many challenging problems on affine $n$-space; see also the overview article 13 .

The present paper deals with a class of affine algebraic manifolds which is well known in affine algebraic geometry but has received relatively little attention from the complex analytic point of view. It is the class of Danielewski surfaces, which are hypersurfaces in $\mathbb{C}_{(x, y, z)}^{3}$ defined by $D_{p}:=\{x y=p(z)\}$, where $p \in \mathbb{C}[z]$ is a polynomial of degree at least 2 and with simple zeros (if the degree of $p$ equals 1 the Danielewski surface is obviously just $\mathbb{C}^{2}$ ). They have been introduced by Danielewski (see 6]) in connection with the cancellation property. Namely, the

Received by the editors April 22, 2010 and, in revised form, April 29, 2010 and September 6, 2010 .

2010 Mathematics Subject Classification. Primary 32Q28; Secondary 32M17.

Key words and phrases. Danielewski surfaces, holomorphic automorphisms, overshears.

(C)2011 American Mathematical Society

Reverts to public domain 28 years from publication 
surfaces have the following remarkable property: If we denote by $D_{p}^{n}$ the hypersurfaces in $\mathbb{C}_{(x, y, z)}^{3}$ defined by $D_{p}^{n}:=\left\{x^{n} y=p(z)\right\}$, then these manifolds, for different $n$, are not homeomorphic, [8], but their products with the affine line $\mathbb{C}$ are algebraically isomorphic. Their polynomial automorphism groups have been determined by Makar-Limanov [16] and [17]. It turns out that the polynomial automorphism groups of $D_{p}^{n}$ are small for $n \geq 2$, but for $n=1$ their automorphism groups are very similar to the polynomial automorphism group of $\mathbb{C}^{2}$.

In this paper we concentrate on the latter ones with $n=1$ and show that it is possible to define a notion of shears and overshears on these surfaces whose behavior is analogous to the behavior of the overshears on $\mathbb{C}^{2}$. Our main result is the following generalization of the main result of Andersén-Lempert theory of $\mathbb{C}^{n}$ to Danielewski surfaces

Theorem 1.1. The group $\operatorname{OS}\left(D_{p}\right)$ generated by overshears is dense in the path component of the identity of the holomorphic automorphism group $\operatorname{Aut}\left(D_{p}\right)$ of a Danielewski surface $D_{p}$.

It is already known that all $D_{p}$ have the density property (see [1], Theorem 1). In that proof so-called hyperbolic vector fields are used, which in the case of $\mathbb{C}^{2}$ are known to be (and so far are the only known) concrete examples of holomorphic automorphisms which are not contained in the group generated by overshears (see [15, [3] and [4]). Thus the main part of our proof consists in proving the fact that the corresponding hyperbolic fields are contained in the Lie algebra generated by overshear fields; see Proposition 3.9.

This paper is organized as follows: In section 2, we define shears and overshears on Danielewski surfaces and recall some important results from 11 which will be used further on. In section 3, we construct some explicit Lie brackets of overshear fields which lead to certain hyperbolic fields. These are used to formulate the missing part in the proof of the main theorem as a purely algebraic problem. In the same section, 3, we solve this problem. The proof of the main theorem comes in section 4. In the last section, section 5 , we comment on the number of connected components of $\operatorname{Aut}\left(D_{p}\right)$ and formulate two questions.

In a forthcoming paper the authors will prove a structure theorem for the group $\operatorname{OS}\left(D_{p}\right)$ in the spirit of the classical van-der Kulk-Jung theorem and analogously to the structure theorem for the algebraic automorphism group of $D_{p}$ indicated by Makar-Limanov in [16. A similar result for $\mathbb{C}^{2}$ has been proven by Ahern and Rudin in [2].

\section{THE DEFINITION OF SHEARS AND OVERSHEARS}

Recall that a holomorphic vector field $V \in \operatorname{VF}_{\text {hol }}(X)$, on a complex manifold $X$, is complete (or globally integrable) if for any initial value $z \in X$ there is a global holomorphic solution of the ordinary differential equation

$$
\dot{\gamma}(t)=V(\gamma(t)), \quad \gamma(0)=z .
$$

In this case, the phase flow (i.e. the map $\mathbb{C} \times X \rightarrow X$ given by $(t, z) \mapsto \gamma_{z}(t)$ ) is a holomorphic action of the additive group $\mathbb{C}_{+}$on $X$, where the subscript $z$ in $\gamma_{z}$ denotes the dependence on the initial value. It is worth mentioning that this action is not necessarily algebraic in the case of an algebraic vector field $V \in \mathrm{VF}_{\text {alg }}(X)$ on an affine algebraic manifold $X$. 
We will consider globally integrable holomorphic vector fields on Danielewski surfaces of a very special form, called shear and overshear fields. There are more globally integrable holomorphic vector fields on Danielewski surfaces. To determine all of them (say up to conjugation by holomorphic automorphisms) is a very difficult task. Even in the simplest case, $\mathbb{C}^{2}$, this seems almost out of reach. It is for instance not known whether a globally integrable holomorphic vector field on $\mathbb{C}^{2}$ can have more than one isolated zero.

From now on let $p \in \mathbb{C}[z]$ be a polynomial with simple zeros. Consider the Danielewski surface

$$
D_{p}=\left\{(x, y, z) \in \mathbb{C}^{3}: x y=p(z)\right\} .
$$

Since $p$ has only simple zeros, the surface $D_{p}$ is smooth and is therefore a smooth affine algebraic hypersurface in $\mathbb{C}^{3}$. Furthermore, $D_{p}$ is an affine modification of $\mathbb{C}_{x, z}^{2}$ along the divisor $D \cong \mathbb{C}=\{x=0\}$ with center $C_{x}=\{x=0, p(z)=0\}$. By symmetry in $x$ and $y$ it is also an affine modification of $\mathbb{C}_{y, z}^{2}$ along the divisor $D \cong \mathbb{C}=\{y=0\}$ with center $C_{y}=\{y=0, p(z)=0\}$. Thus $D_{p}$ contains two copies of $\mathbb{C}^{2} \backslash D \cong \mathbb{C} \times \mathbb{C}^{*}$ as Zariski open subsets. Our definition of shears and overshears on $D_{p}$ will be such that we consider shears and overshears on these subsets (defined as usual shears and overshears on $\mathbb{C} \times \mathbb{C}^{*}$ ) and impose conditions on the functions involved so that our shears and overshears extend holomorphically (not only meromorphically) to $D_{p}$ :

Consider $V=z f(x) \frac{\partial}{\partial z}, f \in \mathcal{O}(\mathbb{C})$, which is an overshear field on $\mathbb{C}_{x}^{*} \times \mathbb{C}_{z}$. Define a biholomorphic mapping $\pi: \mathbb{C}^{*} \times \mathbb{C} \rightarrow D_{p}^{*}:=D_{p} \backslash\{x=0\}$ by

$$
\pi(x, z)=\left(x, \frac{p(z)}{x}, z\right) .
$$

Pushing forward $V$ to $D_{p}^{*}$ via $\pi$ gives us a complete vector field on $D_{p}^{*}$, since $V$ is complete and $\pi$ is biholomorphic. Determine the flow $\varphi_{t}$ of $\pi_{*}(V)$, and consider the time-one map $\varphi_{1}$. This is now a mapping $D_{p}^{*} \rightarrow D_{p}^{*}$. It extends to a mapping $\varphi_{1}$ on $D_{p}$ iff $f(0)=0$. Thus we have to consider overshear fields of the form $V=z x f(x) \frac{\partial}{\partial z}$, and these give us the definition of an overshear on $D_{p}$ :

$$
O_{f}(x, y, z)=\left(x, y+\frac{p\left(z e^{x f(x)}\right)-p(z)}{x}, z e^{x f(x)}\right), f \in \mathcal{O}(\mathbb{C}) .
$$

The same procedure with the shear field $x f(x) \frac{\partial}{\partial z}$ (also here the corresponding coefficient is divisible by $x$ to make the field extend holomorphically to $D_{p}$ ) produces a shear on $D_{p}$ defined by

$$
S_{f}(x, y, z)=\left(x, y+\frac{p(z+x f(x))-p(z)}{x}, z+x f(x)\right), f \in \mathcal{O}(\mathbb{C}) .
$$

Remark 1. Polynomial shears on $D_{p}$ have been considered previously by MakarLimanov [16], where he determined the polynomial automorphism group of $D_{p}$ in terms of generators. In that paper, polynomial shears are called triangular mappings and correspond to our $S_{f}$ in the case where $f \in \mathbb{C}[z]$ is a polynomial. They are time-one maps of locally nilpotent derivations. Our shears can thus be viewed as time-one maps of "locally nilpotent derivations with holomorphic coefficients". Other automorphisms that participate in the generation of the

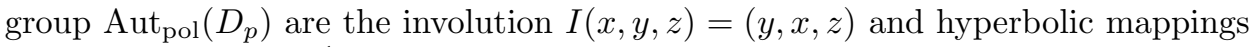
$H_{\lambda}(x, y, z)=\left(\lambda x, \lambda^{-1} y, z\right)$, where $\lambda \in \mathbb{C}^{*}$. 
Remark 2. In the holomorphic case, we have more hyperbolic mappings. In fact, if $f \in \mathcal{O}(\mathbb{C})$ is an arbitrary holomorphic function of one variable, then we have hyperbolic mappings of the form $H_{f}(x, y, z)=\left(x e^{f(z)}, y e^{-f(z)}, z\right)$. These $H_{f}$ are holomorphic automorphisms of $D_{p}$. We will show in a forthcoming paper that these maps are not contained in the overshear group $\operatorname{OS}\left(D_{p}\right)$ (see the definition below), in spite of the fact that their corresponding vector fields are Lie combinations of the shears fields, as we prove in Proposition 3.9 below. There is no contradiction between these two facts, since being a Lie combination of shear fields implies an approximation result in the compact-open topology only.

As mentioned before, there is a second way to view $D_{p}$ as an affine modification of $\mathbb{C}^{2}$, namely, by interchanging $x$ and $y$. This interchange leads to maps of the form $I O_{f}, I$, where $I$ is the involution automorphism from Remark 1 above.

Definition 2.1. The overshear group $\operatorname{OS}\left(D_{p}\right)$ on the Danielewski surface $D_{p}$ is the group generated by the maps $O_{f}, S_{g}$ and $I O_{f} I, I S_{g} I$, where $f, g \in \mathcal{O}(\mathbb{C})$ are arbitrary holomorphic functions of one variable and $I$ is the involution $I(x, y, z)=$ $(y, x, z)$.

Remark 3. If $p$ is a linear polynomial, e.g. $p(z)=z$, the Danielewski surface $D_{p}$ is isomorphic to $\mathbb{C}^{2}$ with coordinates $x$ and $y$. The shears and overshears on $D_{p}$ defined in equations (2.2) and (2.3) are in this case exactly the usual shears and overshears on $\mathbb{C}^{2}$ along the coordinate lines. The group $\operatorname{OS}\left(D_{p}\right)$ in this case is exactly that used by Andersén and Lempert, and thus our main result includes their ground-breaking result from 4 .

We will use the following notation for the vector fields whose time-one maps are our shears and overshears:

$(V 1) O F_{i}^{x}:=z p^{\prime}(z) x^{i} \frac{\partial}{\partial y}+z x^{i+1} \frac{\partial}{\partial z}$

(Overshear field)

(V2) $O F_{i}^{y}:=z p^{\prime}(z) y^{i} \frac{\partial}{\partial x}+z y^{i+1} \frac{\partial}{\partial z}$

(Overshear field)

(V3) $S F_{i}^{x}:=p^{\prime}(z) x^{i} \frac{\partial}{\partial y}+x^{i+1} \frac{\partial}{\partial z}$

(Shear field)

(V4) $S F_{i}^{y}:=p^{\prime}(z) y^{i} \frac{\partial}{\partial x}+y^{i+1} \frac{\partial}{\partial z}$

(Shear field)

(V5) $H F_{f}:=f(z)\left(x \frac{\partial}{\partial y}-y \frac{\partial}{\partial z}\right)$

(Hyperbolic field)

where $i \geq 0$ is an integer and $f \in \mathbb{C}[z]$.

For simplicity of notation we will write $S F^{x}$ and $S F^{y}$ for $S F_{0}^{x}$ and $S F_{0}^{y}$ respectively from now on. The same notation applies to overshear fields.

The algebraic density property for Danielewski surfaces was established by Kaliman and the second author in [11; see Proposition 2.3. For the convenience of the reader let us recall the definition due to Varolin:

Definition 2.2. A complex manifold $X$ has the density property if, in the compactopen topology, the Lie algebra $\operatorname{Lie}_{\text {hol }}(X)$ generated by globally integrable holomorphic vector fields on $X$ is dense in the Lie algebra $\operatorname{VF}_{\text {hol }}(X)$ of all holomorphic vector fields on $X$. An affine algebraic manifold has the algebraic density property if the Lie algebra $\operatorname{Lie}_{\text {alg }}(X)$ generated by globally integrable algebraic vector fields on $X$ coincides with the Lie algebra $\mathrm{VF}_{\text {alg }}(X)$ of all algebraic vector fields on $X$. 
Clearly the algebraic density property implies the density property for any affine algebraic manifold $X$. Indeed, since the tangent sheaf of an affine algebraic manifold $X$ can be generated by a finite number of global algebraic sections, Oka-Weill approximation shows that the algebraic density property for such $X$ implies the density property for $X$; in particular, this holds for Danielewski surfaces.

In [11] the following is proven (see Proposition 2.3 and its proof):

Proposition 2.3. $D_{p}$ has the algebraic density property. More precisely: Any algebraic vector field on $D_{p}$ is a Lie combination of (finitely many) vector fields of the form $(V 1)-(V 5)$ above, i.e., of shear fields, overshear fields and hyperbolic fields.

Corollary 2.4. If we can write any hyperbolic vector field as a Lie combination of shear and overshear vector fields, then any algebraic vector field can be written as a Lie combination of shear and overshear vector fields.

\section{Hyperbolic Fields ARE Lie combinations of SHEARS}

In this section we will prove that all hyperbolic fields $H F_{f}, f \in \mathbb{C}[z]$, are Lie combinations of the fields $S F_{i}^{x}, S F_{i}^{y}, O F_{i}^{x}, O F_{i}^{y}$.

Straightforward calculations show:

Lemma 3.1. For every integer $i \geq 0$,

$$
\begin{aligned}
& \text { (a) }\left[S F_{i}^{x}, S F_{i}^{y}\right]=H F_{\left(p^{i+1}\right)^{\prime \prime} /(i+1)}, \\
& \text { (b) }\left[S F_{i}^{x}, O F_{i}^{y}\right]-\left[S F_{i}^{y}, O F_{i}^{x}\right]=H F_{2 z\left(p^{i+1}\right)^{\prime \prime} /(i+1)+p^{\prime} p^{i}}, \\
& \text { (c) }\left[O F_{i}^{x}, O F_{i}^{y}\right]=H F_{\left(z^{2}\left(p^{i+1}\right)^{\prime \prime}+z\left(p^{i+1}\right)^{\prime}\right) /(i+1) .}
\end{aligned}
$$

To make calculations for more sophisticated brackets easier, let us remember from [11] that there is a unique (up to a constant) algebraic volume form on $\omega$ on $D_{p}$. For every volume-preserving (algebraic) vector field, $\theta$ holds (since $\omega$ is a closed form):

$$
0=L_{\omega}(\theta)=d i_{\theta} \omega+i_{\theta} d \omega=d i_{\theta} \omega
$$

Since $D_{p}$ is simply connected we get $i_{\theta} \omega=d f$ for some function $f \in \mathbb{C}\left[D_{p}\right]$. Thus we have a bijection between algebraic volume preserving fields and polynomial functions modulo constants.

Shear fields and hyperbolic fields are volume preserving. Using the fact that in the coordinates $(x, z) \in \mathbb{C}^{*} \times \mathbb{C}$ the form $\omega$ equals $\frac{d x \wedge d z}{x}$ (thus this expression extends regularly to $D_{p}$ from $\left.\mathbb{C} \times \mathbb{C}^{*}\right)$, one easily calculates the functions corresponding to shear fields and hyperbolic fields:

Lemma 3.2. The following holds for all $f \in \mathcal{O}(\mathbb{C})$ :

$$
i_{S F^{x}} \omega=-d x, \quad i_{S F^{y}} \omega=d y, \quad i_{H F_{f^{\prime}(z)}} \omega=d f(z) .
$$

The following is useful for calculations of brackets.

Lemma 3.3. If $\theta$ is a volume-preserving field with $i_{\theta} \omega=d f$ and $\psi$ is another volume preserving field, then

$$
i_{[\psi, \theta]} \omega=d L_{\psi}(f) .
$$

The following lemma produces our important Lie combinations. 
Lemma 3.4. If $f_{1}, f_{2}, \ldots, f_{k}$ are finitely many holomorphic functions of the variable $z$, then

$$
\begin{aligned}
& \text { (1a) }\left[H F_{f_{k}},\left[H F_{f_{k-1}},\left[\ldots,\left[H F_{f_{1}}, S F^{x}\right]\right] \ldots\right]=-x H F_{\left(\prod f_{\alpha}\right)^{\prime}}+\left(\prod f_{\alpha}\right) S F^{x}\right. \text {, } \\
& \text { (1b) }\left[H F_{f_{k}},\left[H F_{f_{k-1}},\left[\ldots,\left[H F_{f_{1}}, S F^{y}\right]\right] \ldots\right]=(-1)^{k}\left\{y H F_{\left(\prod f_{\alpha}\right)^{\prime}}+\left(\prod f_{\alpha}\right) S F^{y}\right\}\right. \text {, } \\
& \text { (2a) }\left[S F^{y},\left[H F_{f_{k}},\left[H F_{f_{k-1}},\left[\ldots,\left[H F_{f_{1}}, S F^{x}\right]\right] \ldots\right]=H F_{-\left(p \prod f_{\alpha}\right)^{\prime \prime}}\right. \text {, }\right. \\
& \text { (2b) }\left[S F^{x},\left[H F_{f_{k}},\left[H F_{f_{k-1}},\left[\ldots,\left[H F_{f_{1}}, S F^{y}\right]\right] \ldots\right]=H F_{(-1)^{k}\left(p \prod f_{\alpha}\right)^{\prime \prime}}\right. \text {, }\right. \\
& \text { (3a) }\left[S F^{y},\left[H F_{f_{k}},\left[H F_{f_{k-1}},\left[\ldots,\left[H F_{f_{1}}, O F^{x}\right]\right] \ldots\right]=H F_{-z\left(p \prod f_{\alpha}\right)^{\prime \prime}-p\left(\prod f_{\alpha}\right)^{\prime}}\right.\right. \\
& +y\left(\prod f_{\alpha}\right) S F^{x} \\
& \text { (3b) }\left[S F^{x},\left[H F_{f_{k}},\left[H F_{f_{k-1}},\left[\ldots,\left[H F_{f_{1}}, O F^{y}\right]\right] \ldots\right]\right.\right. \\
& =H F_{(-1)^{k} z\left(p \prod f_{\alpha}\right)^{\prime \prime}+(-1)^{k} p\left(\prod f_{\alpha}\right)^{\prime}}+(-1)^{k} x\left(\prod f_{\alpha}\right) S F^{y}, \\
& \text { (3c) }(-1)^{k}\left[S F^{x},\left[H F_{f_{k}},\left[H F_{f_{k-1}},\left[\ldots,\left[H F_{f_{1}}, O F^{y}\right]\right] \ldots\right]\right.\right. \\
& -\left[S F^{y},\left[H F_{f_{k}},\left[H F_{f_{k-1}},\left[\ldots,\left[H F_{f_{1}}, O F^{x}\right]\right] \ldots\right]\right.\right.
\end{aligned}
$$

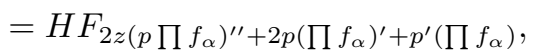

$$
\begin{aligned}
& \text { (4) }\left[O F^{y},\left[H F_{f_{k}},\left[H F_{f_{k-1}},\left[\ldots,\left[H F_{f_{1}}, O F^{x}\right]\right] \ldots\right]=H F_{-z^{2}\left(p \prod f_{\alpha}\right)^{\prime \prime}-z\left(p \prod f_{\alpha}\right)^{\prime}}\right. \text {, }\right. \\
& \text { (5) }(-1)^{k}\left[O F^{x},\left[H F_{f_{k}},\left[H F_{f_{k-1}},\left[\ldots,\left[H F_{f_{1}}, S F^{y}\right]\right] \ldots\right]\right.\right. \\
& -\left[O F^{y},\left[H F_{f_{k}},\left[H F_{f_{k-1}},\left[\ldots,\left[H F_{f_{1}}, S F^{x}\right]\right] \ldots\right]=H F_{2 z\left(p \prod f_{\alpha}\right)^{\prime \prime}+p^{\prime}\left(\prod f_{\alpha}\right)} .\right.\right.
\end{aligned}
$$

Proof. (1) By Lemma 3.2 we have $i_{S F^{x}} \omega=-d x$. Denote by $\beta$ the left hand side of (1a). Lemma 3.3 gives

$$
\begin{gathered}
i_{\beta} \omega=-d\left(L_{H F_{f_{k}}} \ldots L_{H F_{f_{1}}}(x)\right)=-d\left(f_{k}(z) \ldots f_{1}(z) x\right) \\
=-x d\left(\prod f_{\alpha}\right)-\left(\prod f_{\alpha}\right) d x .
\end{gathered}
$$

Thus by Lemma 3.2, $\beta=-x H F_{\left(\prod f_{\alpha}\right)^{\prime}}+\left(\prod f_{\alpha}\right) S F^{x}$, which is (1a). The proof of (1b) is similar.

(2) Denote by $\gamma$ the left hand side of (2a). By the same logic as above

$$
\begin{aligned}
i_{\gamma} \omega= & -d\left(L_{S F^{y}} \cdot L_{H F_{f_{k}}} \ldots L_{H F_{f_{1}}}(x)\right)=-d\left(L_{S F^{y}}\left(x\left(\prod f_{\alpha}\right)\right)\right) \\
& =-d\left(x y\left(\prod f_{\alpha}\right)^{\prime}+p^{\prime}\left(\prod f_{\alpha}\right)\right)=-d\left(p \prod f_{\alpha}\right)^{\prime}
\end{aligned}
$$

using the fact that $x y=p$. Again Lemma 3.2 yields $\gamma=H F_{-\left(p \prod f_{\alpha}\right)^{\prime \prime}}$, which is (2a). The proof of $(2 \mathrm{~b})$ is similar.

(3) Since $O F^{x}=z S F^{x}$ and the function $z$ is in the kernel of hyperbolic fields, we get

$$
\begin{aligned}
& {\left[S F^{y},\left[H F_{f_{k}},\left[H F_{f_{k-1}},\left[\ldots,\left[H F_{f_{1}}, O F^{x}\right]\right] \ldots\right]\right.\right.} \\
= & {\left[S F^{y}, z\left[H F_{f_{k}},\left[H F_{f_{k-1}},\left[\ldots,\left[H F_{f_{1}}, S F^{x}\right]\right] \ldots\right] .\right.\right.}
\end{aligned}
$$

Using (2a) and (1a) the above equation equals

$$
\begin{aligned}
& z H F_{-\left(p \prod f_{\alpha}\right)^{\prime \prime}}+S F^{y}(z)\left(-x H F_{\left(\prod f_{\alpha}\right)^{\prime}}+\left(\prod f_{\alpha}\right) S F^{x}\right) \\
& =H F_{-z\left(p \prod f_{\alpha}\right)^{\prime \prime}}+y\left(-x H F_{\left(\prod f_{\alpha}\right)^{\prime}}+\left(\prod f_{\alpha}\right) S F^{x}\right),
\end{aligned}
$$

which using $p=x y$ again is (3a). (3b) is proved the same way. (3c) follows immediately from (3a) and (3b) using the fact that $x S F^{y}-y S F^{x}=H F_{p^{\prime}}$. 
(4) Since $z$ is in the kernel of all hyperbolic fields, we get

$$
\begin{aligned}
& {\left[O F^{y},\left[H F_{f_{k}},\left[H F_{f_{k-1}},\left[\ldots,\left[H F_{f_{1}}, O F^{x}\right]\right] \ldots\right]\right.\right.} \\
= & {\left[z S F^{y}, z\left[H F_{f_{k}},\left[H F_{f_{k-1}},\left[\ldots,\left[H F_{f_{1}}, S F^{x}\right]\right] \ldots\right]\right.\right.} \\
= & z^{2}\left[S F^{y},\left[H F_{f_{k}},\left[H F_{f_{k-1}},\left[\ldots,\left[H F_{f_{1}}, S F^{x}\right]\right] \ldots\right]\right.\right. \\
+ & z S F^{y}(z)\left[H F_{f_{k}},\left[H F_{f_{k-1}},\left[\ldots,\left[H F_{f_{1}}, S F^{x}\right]\right] \ldots\right]\right. \\
- & z\left[H F_{f_{k}},\left[H F_{f_{k-1}},\left[\ldots,\left[H F_{f_{1}}, S F^{x}\right]\right] \ldots\right](z) S F^{y} .\right.
\end{aligned}
$$

Now one applies (1a) and finally uses the fact that $x S F^{y}-y S F^{x}=H F_{p^{\prime}}$ to get (4).

(5) is proved in the same manner as (3); we leave it as an exercise for the reader.

These brackets give us concrete ways to produce hyperbolic fields as Lie combinations of shear and overshear fields. We can now reformulate our problem using Lemma 3.1 and Lemma 3.4 (2a), (3c), (4) and (5) subtracted from (3c) as follows:

Let $W \subset \mathbb{C}[z]$ be a vector subspace such that

(C1) (i) $\left(p^{i+1}\right)^{\prime \prime},(i i) 2 z\left(p^{i+1}\right)^{\prime \prime}+\left(p^{i+1}\right)^{\prime}$, and (iii) $z^{2}\left(p^{i+1}\right)^{\prime \prime}+z\left(p^{i+1}\right)^{\prime} \in W$ for each integer $i \geq 0$.

If finitely many functions $f_{1}, f_{2}, \ldots, f_{k} \in W$, then

(C2) $\left(p \prod_{\alpha=1}^{k} f_{\alpha}\right)^{\prime \prime} \in W$,

(C3) $2 z\left(p \prod f_{\alpha}\right)^{\prime \prime}+2 p\left(\prod f_{\alpha}\right)^{\prime}+p^{\prime}\left(\prod f_{\alpha}\right) \in W$

(C4) $z^{2}\left(p \prod f_{\alpha}\right)^{\prime \prime}+z\left(p \prod f_{\alpha}\right)^{\prime} \in W$ and

(C5) $p\left(\prod f_{\alpha}\right)^{\prime} \in W$.

Show that $W=\mathbb{C}[z]$.

The strategy of our proof is first to show that the algebra $\mathcal{A}_{W}$ generated by the elements of a vector subspace $W$ satisfying properties $(C 1)$ to $(C 5)$ is equal to $\mathbb{C}[z]$.

Lemma 3.5. There is no point $a \in \mathbb{C}$ such that all elements in $\mathcal{A}_{W}$ have a common zero at $a$.

Proof. Suppose to the contrary that such a point $a$ exists. Then $p^{\prime \prime}(a)=0$, from $(C 1)(i)$. Furthermore, using $(C 1)(i i)$, we get $2 a p^{\prime \prime}(a)+p^{\prime}(a)=0$. Thus $p^{\prime}(a)=0$. If the degree of $p$ is one, this yields a contradiction. From now on let $n \geq 2$ be the degree of $p$. Since $p$ and $p^{\prime}$ have no common zeros, $p(a) \neq 0$. Define inductively functions $f_{k} \in \mathbb{C}[z]$ by $f_{0}=p^{\prime \prime}$ and $f_{k+1}=p f_{k}^{\prime}$. By (C5) they are in $W$. Since $p(a) \neq 0$ we have $f_{k}^{\prime}(a)=0$ for all $k$. Using $f_{k}^{\prime}=p^{\prime} f_{k-1}^{\prime}+p f_{k-1}^{\prime \prime}$ we find $f_{k-1}^{\prime \prime}(a)=0$ for all $k$. Continuing by induction we find that all derivatives of the functions $f_{k}$ at the point $a$ are zero, which is absurd since $f_{0}^{(n-2)}=p^{(n)}$ is a nonzero constant.

Lemma 3.6. There is no point $b \in \mathbb{C}$ such that the derivatives of all elements in $\mathcal{A}_{W}$ have a common zero at $b$.

Proof. Assume that such $b$ exists. Since $p^{\prime \prime} \in W$ by $(C 1)(i)$, we get that $p^{\prime \prime \prime}(b)=0$. From $(C 1)(i i)$ we get $\left(2 z p^{\prime \prime}(z)+p^{\prime}(z)\right)^{\prime}(b)=0$, which implies that $p^{\prime \prime}(b)=0$. Furthermore, $(C 1)($ iii $)$ gives that $\left(z^{2} p^{\prime \prime}(z)+z p^{\prime}(z)\right)^{\prime}(b)=0$, which implies that $p^{\prime}(b)=0$. Thus $p(b) \neq 0$, since $p$ only has simple zeros. Define again inductively functions $f_{k} \in \mathbb{C}[z]$ by $f_{0}=p^{\prime \prime}$ and $f_{k+1}=p f_{k}^{\prime}$. By $(C 5)$ they are in $W$. Since $p(b) \neq 0$ and $f_{k}^{\prime}=p^{\prime} f_{k-1}^{\prime}+p f_{(k-1)}^{\prime \prime}$, we find $f_{k-1}^{\prime \prime}(b)=0$ for all $k$. 
Now $f_{k}^{\prime \prime}=p^{\prime} f_{k-1}^{\prime \prime}+p f_{k-1}^{\prime \prime \prime}+p^{\prime \prime} f_{k-1}^{\prime}$, so again $p(b) \neq 0$ implies $f_{k-1}^{\prime \prime \prime}(b)=0$ for all $k$. Continuing inductively with higher derivatives we find that all derivatives of the functions $f_{k}$ at the point $b$ are zero, which is a contradiction since $f_{0}^{(n-2)}=p^{(n)}$ is a nonzero constant.

Lemma 3.7. There is no pair of points $c_{1} \neq c_{2} \in \mathbb{C}$ such that all elements of $\mathcal{A}_{W}$ take the same values at $c_{1}$ and $c_{2}$.

Proof. Suppose to the contrary that such a pair of points exists.

Step 1:

By $(C 1)(i)$ we have $p^{\prime \prime}\left(c_{1}\right)=p^{\prime \prime}\left(c_{2}\right)=: A$. We get from $(C 1)(i i),(i i i)$ for $i=0$ that

$$
\begin{array}{r}
2\left(c_{1}-c_{2}\right) A=p^{\prime}\left(c_{2}\right)-p^{\prime}\left(c_{1}\right), \\
\left(c_{1}^{2}-c_{2}^{2}\right) A=c_{2} p^{\prime}\left(c_{2}\right)-c_{1} p^{\prime}\left(c_{1}\right) .
\end{array}
$$

Eliminating $A$ from these equations we find $\left(c_{1}-c_{2}\right)\left(p^{\prime}\left(c_{1}\right)+p^{\prime}\left(c_{2}\right)\right)=0$. Hence $-p^{\prime}\left(c_{1}\right)=p^{\prime}\left(c_{2}\right)$, and using this fact in the first equation yields

$$
p^{\prime}\left(c_{2}\right)=A\left(c_{1}-c_{2}\right) .
$$

Step 2:

Suppose for a moment that $A \neq 0$. Property $(C 1)(i)$ with $i=1$ implies that $\left(p^{2}\right)^{\prime \prime}=2\left(p^{\prime}\right)^{2}+2 p p^{\prime \prime}$ takes the same value $B$ at $c_{1}, c_{2}$. Thus $p\left(c_{1}\right)=p\left(c_{2}\right)$ by virtue of $A \neq 0$. Using $(C 1)(i i)$ with $i=1: 2 c_{1} B+p\left(c_{1}\right) p^{\prime}\left(c_{1}\right)=2 c_{2} B+p\left(c_{2}\right) p^{\prime}\left(c_{2}\right)$; thus $2 B\left(c_{1}-c_{2}\right)=2 p^{\prime}\left(c_{2}\right)\left(p\left(c_{1}\right)+p\left(c_{2}\right)\right)=2 A\left(c_{1}-c_{2}\right) 2 p\left(c_{2}\right)$ and thus $B=2 A p\left(c_{2}\right)$. Remembering the definitions of $A$ and $B$, this yields $2 p p^{\prime \prime}+2\left(p^{\prime}\right)^{2}=2 p^{\prime \prime} p$ at $c_{2}$. Thus $p^{\prime}\left(c_{2}\right)=0$, which implies that $A=0$. Thus our assumption that $A \neq 0$ led to $A=0$ and therefore $p^{\prime \prime}\left(c_{1}\right)=p^{\prime \prime}\left(c_{2}\right)=p^{\prime}\left(c_{1}\right)=p^{\prime}\left(c_{2}\right)=0$.

Step 3:

As in Step 1 we use the fact (following from $(C 2),(C 3)$ minus $(C 5),(C 4)$ respectively) that for any $f \in W$ the functions

$$
(p f)^{\prime \prime}, \quad 2 z(p f)^{\prime \prime}+(p f)^{\prime}, \quad z^{2}(p f)^{\prime \prime}+z(p f)^{\prime}
$$

take the same values at $c_{1}, c_{2}$ to conclude that $(p f)^{\prime}\left(c_{1}\right)=-(p f)^{\prime}\left(c_{2}\right)$ for all $f \in W$. Since $p^{\prime}$ vanishes at these points we get $p\left(c_{1}\right) f^{\prime}\left(c_{1}\right)=-p\left(c_{2}\right) f^{\prime}\left(c_{2}\right)$. By $(C 5)$ $p\left(c_{1}\right) f^{\prime}\left(c_{1}\right)=p\left(c_{2}\right) f^{\prime}\left(c_{2}\right)$. Thus for all $f \in W$ there holds $p\left(c_{1}\right) f^{\prime}\left(c_{1}\right)=0$. By Lemma 3.6 this implies $p\left(c_{1}\right)=0$, contradicting $p^{\prime}\left(c_{1}\right)=0$.

Proposition 3.8. The subalgebra $\mathcal{A}_{W}$ of $\mathbb{C}[z]$ generated by $W$ coincides with $\mathbb{C}[z]$.

Proof. Lemmas 3.6 and 3.7 yield the existence of a finite number of polynomials $q_{1}, q_{2}, \ldots, q_{K} \in \mathcal{A}_{W}$ such that the map $\mathbb{C} \rightarrow \mathbb{C}^{K}$ given by

$$
z \mapsto\left(\left(q_{1}(z), q_{2}(z), \ldots, q_{K}(z)\right)\right)
$$

is an injective immersive embedding. Now the subalgebra generated by $q_{1}, q_{2}, \ldots$, $q_{K}$ and constants is isomorphic to $\mathbb{C}[z]$. Thus the subalgebra $\mathcal{A}_{0}$ generated by $q_{1}, q_{2}, \ldots, q_{K}$ (without constants) is an (in view of Lemma 3.6 maximal) ideal in $\mathbb{C}[z]$. Lemma 3.5 implies that $\mathcal{A}_{0}=\mathbb{C}[z]$. Since by construction $\mathcal{A}_{0}$ contains $\mathcal{A}_{W}$, the proof is complete.

Proposition 3.9. $W=\mathbb{C}[z]$. 
Proof. Since by Proposition 3.8 the subalgebra generated by elements of $W$ is the whole polynomial ring, we get by $(C 2)-(C 5)$ that the polynomials $(p g)^{\prime \prime}, 2 z(p g)^{\prime \prime}+$ $2 p(g)^{\prime}+p^{\prime}(g), z^{2}(p g)^{\prime \prime}+z(p g)^{\prime}, p g^{\prime}$ are continued in $W$ for all $g \in \mathbb{C}[z]$.

Looking at polynomials of the same degree $k+n-2$, the following polynomials (for different $g$ being powers of $z$ ) are contained in $W$ :

$$
\left(z^{k} p\right)^{\prime \prime}, \quad z^{2}\left(z^{(k-2)} p\right)^{\prime \prime}+z\left(z^{(k-2)} p\right)^{\prime}, \quad z^{(k-2)} p .
$$

The above polynomials are all linear combinations of the three polynomials $z^{(k-2)} p, z^{(k-1)} p^{\prime}$ and $z^{k} p^{\prime \prime}$. Since the matrix of coefficients

$$
\left[\begin{array}{ccc}
k(k-1) & 2 k & 1 \\
(k-2)^{2} & 2 k-3 & 1 \\
1 & 0 & 0
\end{array}\right]
$$

has determinant 3 , the polynomials $z^{k-2} p, z^{k-1} p^{\prime}$ and $z^{k} p^{\prime \prime}$ are in $W$. This means (if we do it for all $k$ ) that the principal ideal $(p)$ generated by $p$ and the principal ideals $\left(p^{\prime}\right)$ and $\left(p^{\prime \prime}\right)$ are contained in $W$. Since $p$ and $p^{\prime}$ have no common zeros, the Nullstellensatz implies that $W=\mathbb{C}[z]$.

Thus we have an immediate consequence of Corollary 2.4 and Proposition 3.9, which is the core part of our main theorem:

Theorem 3.10. Let $D_{p}$ be the Danielewski surface with respect to a polynomial $p \in \mathbb{C}[z]$ with simple zeros. Then the Lie algebra generated by overshears equals the Lie algebra of all algebraic vector fields on $D_{p}$.

\section{The Density of The OVERSheAR Group}

To make the presentation self-contained we outline the proof of a version of the Andersén-Lempert theorem, Proposition 4.2 (see also Forstnerič-Rosay's Theorem 1.1 in 9]). Let $V$ be a holomorphic vector field on a complex manifold $M$. Assume that $K_{t}: M \rightarrow M$ is a family of holomorphic mappings which are $\mathcal{C}^{1}$ in $t \in[0,1]$. Assume furthermore that $K_{0}=\mathrm{Id}$ and that $\left.\frac{d K_{t}}{d t}\right|_{t=0}=V$. Such a $K$ is called a consistent algorithm for $V$. Assume that $\varphi_{t}(z)$ is the flow of $V$ starting at $z$. Euler's method gives

$$
\lim _{N \rightarrow \infty} K_{t / N}^{N}(z)=\varphi_{t}(z),
$$

locally uniformly on subsets of $\mathbb{R} \times M$, where either side is defined (see Theorem 2.1.26 in [1]).

Here are two consistent algorithms for the sum and for the Lie bracket of two vector fields; see 21 .

Lemma 4.1. Let $V$ and $W$ be two holomorphic vector fields on $M$ with flows $\left\{\varphi_{t}\right\}$, respectively $\left\{\psi_{t}\right\}$. Then

$$
\begin{aligned}
& \text { 1. }\left.\frac{d}{d t}\right|_{t=0} \varphi_{t} \circ \psi_{t}=V+W, \\
& \text { 2. }\left.\frac{d}{d t}\right|_{t=0^{+}} \psi_{-\sqrt{t}} \circ \varphi_{-\sqrt{t}} \circ \psi_{\sqrt{t}} \circ \varphi_{\sqrt{t}}=[V, W] .
\end{aligned}
$$

Proposition 4.2. Assume that $\Omega \subset D_{p}$ is a domain. Let $\Phi:[0,1] \times \Omega \rightarrow D_{p}$ be a $\mathcal{C}^{1}$-family such that $\Phi_{t}: \Omega \rightarrow D_{p}$ is injective and holomorphic for each fixed 
$t \in[0,1]$. For each time $t_{0} \in[0,1]$ and for $z \in \Phi_{t_{0}}(\Omega)$, define the time dependent vector field $X_{t_{0}}$ by

$$
X_{t_{0}}(z):=\left.\frac{d}{d t}\right|_{t=t_{0}} \Phi_{t}\left(\Phi_{t_{0}}^{-1}(z)\right)
$$

Furthermore, assume that all vector fields $X_{t_{0}}$ for $t_{0} \in[0,1]$ can be approximated (on compacts in their domain of definition) by (globally defined) polynomial vector fields on $D_{p}$.

If $\Phi_{0}$ can be approximated by elements in $\operatorname{OS}\left(D_{p}\right)$, then $\Phi_{1}$ can also be approximated by elements in $\mathrm{OS}\left(D_{p}\right)$.

Remark 4. By approximation, we mean uniform approximation on compact subsets.

Proof of Proposition 4.2. A first observation is that $\Phi_{1} \circ \Phi_{0}^{-1}$ equals the result of integrating $X_{t}$ from 0 to 1 .

Let $\varphi_{s}^{t}$ be the flow of $X_{t}$. Construct a partition of $[0,1]$ by pieces of length $1 / n$. By Euler's method, the flow of $X_{t}$ is approximated by flowing the time independent field $X_{k / n}$ from time $k / n$ to $(k+1) / n$ for $k=0, \ldots, n-1$. Now use Euler's method again to approximate each flow $\varphi_{s}^{k / n}$ for $k=0, \ldots, n$ as follows: By assumption we can approximate $X_{k / n}$ uniformly by polynomial vector fields. Using Theorem 3.10 we can write every polynomial vector field as a Lie combination of complete polynomial vector fields whose flows are in $\operatorname{OS}\left(D_{p}\right)$. Their flows are shear and overshear automorphisms, so we can approximate $\Phi_{1} \circ \Phi_{0}^{-1}$ using Lemma 4.1 by finite compositions of shears and overshears. Hence we can approximate $\Phi_{1}=\left(\Phi_{1} \circ \Phi_{0}^{-1}\right) \circ \Phi_{0}$ by elements in $\operatorname{OS}\left(D_{p}\right)$.

Proof of Theorem 1.1. Let $K \subset D_{p}$ be a compact set, and let $\Psi$ be an arbitrary automorphism in the path component of the identity. We need to show that we can approximate this automorphism on $K$. By assumption there is a continuous path $\Phi:[0,1] \rightarrow \operatorname{Aut}\left(D_{p}\right)$ of automorphisms such that $\Phi_{0}=\operatorname{Id}$ and $\Phi_{1}=\Psi$. This family of mappings induces a mapping, continuous in $t$, defined on $[0,1] \times D_{p} \rightarrow D_{p}$ given by $(t,(x, y, z)) \mapsto \Phi_{t}(x, y, z)$. With some abuse of notation, we will call this mapping $\Phi_{t}$ as well. Using sublevel sets of exhaustion functions we can choose a Runge domain $\Omega \Subset D_{p}$ containing the compact set $\bigcup_{t \in[0,1]} \Phi_{t}(K)$. Furthermore, let $\widetilde{\Omega} \Subset D_{p}$ be an open set such that $\bigcup_{t \in[0,1]} \Phi_{t}(\Omega) \subset \widetilde{\Omega}$. By a convolution of $\Phi_{t}$, we get a smooth mapping $\widetilde{\Phi}_{t}:[0,1] \times D_{p} \rightarrow \mathbb{C}^{3}$. By differentiating under the integral sign we see that $\widetilde{\Phi}_{t}$ is holomorphic for fixed $t$. Then use the tubular neighborhood theorem [7] to project $\widetilde{\Phi}_{t}(z)$ back into the manifold $D_{p}$; i.e. if $N\left(D_{p}\right)$ is the normal bundle of $D_{p}$ in $\mathbb{C}^{3}$ and $\mathcal{N}$ is a neighborhood of the zero section in $N\left(D_{p}\right)$ which is biholomorphic, via $\alpha: \mathcal{N} \rightarrow U$, to an open neighborhood $U$ of $D_{p}$ in $\mathbb{C}^{3}$ then define $\widehat{\Phi}:[0,1] \times D_{p} \rightarrow D_{p}$ by $\widehat{\Phi}_{t}(z)=\pi \circ \alpha^{-1} \circ \widetilde{\Phi}_{t}(z)$, where $\pi$ is the projection. Since injectivity is an open condition in $\mathcal{C}^{1}$-topology and approximation in values for holomorphic mappings automatically implies approximation of the derivatives (in a smaller compactum, by Cauchy's Integral Formula), we may as well assume that $\widehat{\Phi}_{t}: \widetilde{\Omega} \rightarrow D_{p}$ is injective. Thus the vector fields $X_{t}$, from the proof of Proposition 4.2. are well defined on $\Omega$. Since $\Omega$ is Runge we can approximate $X_{t}$ by polynomial vector fields, and now Proposition 4.2 implies that we can approximate $\widehat{\Phi}_{1}$ arbitrarily close by elements in $\operatorname{OS}\left(D_{p}\right)$. Also $\widehat{\Phi}_{1}$ is by construction arbitrarily close to $\Phi_{1}=\Psi$, which concludes the proof. 


\section{ON THE NUMBER OF COMPONENTS OF THE AUTOMORPHISM GROUP}

If the degree of the defining polynomial is one, the Danielewski surface $D_{p}$ is $\mathbb{C}^{2}$ and it's group of holomorphic automorphisms is path-connected. If the degree is at least 2, the situation is different: Since they are contained in a flow, the elements $H_{f}, S_{f}^{x}, S_{f}^{y}, O_{f}^{x}, O_{f}^{y}$ in $\operatorname{Aut}\left(D_{p}\right)$ are contained in the path component of the identity of $\operatorname{Aut}\left(D_{p}\right)$. On the other hand, the map $I(x, y, z)=(y, x, z)$ is not. It can be shown that it acts as $\alpha \mapsto-\alpha$ on $H^{2}\left(D_{p}, \mathbb{Z}\right) \cong \mathbb{Z}^{n-1}$, where $n=\operatorname{deg} p$. Thus $\operatorname{Aut}\left(D_{p}\right)$ has at least two path components.

Remark 5. The authors do not know how to calculate the exact number of (path) components of $\operatorname{Aut}\left(D_{p}\right)$. The results of Makar-Limanov show that the algebraic automorphism group has exactly two (path) components. Moreover, we do not know whether the path-connected component of the identity in $\operatorname{Aut}\left(D_{p}\right)$ is equal to the connected component of the identity in $\operatorname{Aut}\left(D_{p}\right)$. For the diffeomorphism group of a noncompact manifold they may differ (even if the contrary is stated in the literature), as the following example shows.

Example 1. Take a Riemann surface $X$ of infinite genus, say a connected sum of countably many tori $T_{i}, i=1, \ldots, \infty$. We define a diffeomorphism $\varphi_{i}$ of $X$ by being an identity at all tori except the $i$ th, and there we build in the Dehn twist in the following way: We cut the torus open along an $S^{1}$, rotate one side by $2 \pi$ and glue back. This rotation is smoothly cut down to zero rotation a bit from the place where we cut in order to extend it smoothly to the whole surface $X$.

Each of these $\varphi_{i}$ acts nontrivially on the fundamental group, thus does not belong to the path component of the identity. However, in the compact open topology the sequence $\varphi_{i}$ converges to identity since the support of $\varphi_{i}$ is leaving any compact subset for $i \rightarrow \infty$.

We end the paper with two open problems:

Problem 5.1. In [14] it is shown that the Danielewski surfaces $D_{p}$ admit the algebraic volume density property with respect to the unique algebraic volume form $\omega$ on them. Is it true that the group generated by shears is dense in the path component of the group $\operatorname{Aut}^{\omega}\left(D_{p}\right)$ of volume-preserving holomorphic automorphisms of $D_{p}$ ? The results of the present paper combined with the method from [14 do not suffice to prove this, since we use overshear fields (not volume preserving) in the Lie combinations from Lemma 3.4 that produce hyperbolic fields (volume preserving). This problem is also interesting in connection with the following question posed in [5]: If an affine algebraic manifold is flexible (the group generated by locally nilpotent derivations acts transitively on it), does it have the algebraic volume density property?

Problem 5.2. Determine the number of (path) components of the group $\operatorname{Aut}\left(D_{p}\right)$. Are the path components also the connected components? More generally: Let $\operatorname{Aut}(X)$ be the topological group of holomorphic automorphisms of a complex manifold $X$ equipped with compact-open topology. Are the path components of $\operatorname{Aut}(X)$ also the connected components? 


\section{ACKNOWLEDGEMENTS}

The authors thank Harm Derksen, Björn Ivarsson, Shulim Kaliman, Sverre Smalø and Erlend Fornæss Wold for fruitful discussions on the subject. They especially thank the referee for pointing out a mistake in the original version of the paper and for making valuable comments to improve the presentation.

The research of the first author was partially supported by Schweizerische Nationalfonds grant No 200020-124668/1. The research of the second author was supported by Forskarskolan för Matematik och Beräkningsvetenskap FMB.

\section{REFERENCES}

1. R. Abraham, J. E. Marsden, Foundations of mechanics. Second edition, revised and enlarged, Benjamin/Cummings Publishing Co., Inc., Advanced Book Program, Reading, Mass., 1978. MR515141 (81e:58025)

2. P. Ahern, W. Rudin, Periodic automorphisms of $\mathbb{C}^{n}$, Indiana Univ. Math. J. 44 (1995), no. 1, 287-303. MR1336443 (96f:32038)

3. E. Andersén, Volume-preserving automorphisms of $\mathbb{C}^{n}$, Complex Variables Theory Appl. 14 (1990), no. 1-4, 223-235. MR.1048723 (91d:32047)

4. E. Andersén, L. Lempert, On the group of holomorphic automorphisms of $\mathbb{C}^{n}$, Invent. Math. 110 (1992), no. 2, 371-388. MR1185588(93i:32038)

5. I. Arzhantsev, H. Flenner, S. Kaliman, F. Kutzschebauch, M. Zaidenberg, The automorphism group of a flexible affine variety is infinitely transitive (2010), arXiv:1011.5375.

6. W. Danielewski, On a cancellation problem and automorphism groups of affine algebraic varieties, preprint, Warsaw (1989).

7. F. Docquier, H. Grauert, Levisches Problem und Rungescher Satz für Teilgebiete Steinscher Mannigfaltigkeiten, Math. Ann. 140 (1960), 94-123. MR0148939(26:6435)

8. K. H. Fieseler, On complex affine surfaces with $\mathbb{C}^{+}$-action, Comment. Math. Helv. 69 (1994), no. 1, 5-27. MR1259603 (95b:14027)

9. F. Forstnerič, J. P. Rosay, Approximations of biholomorphic mappings by automorphisms of $\mathbb{C}^{n}$, Invent. Math. 112 (1993), no. 2, 323-349. MR1213106 (94f:32032)

10. H. W. E. Jung, Über ganze birationale Transformationen der Ebene, J. Reine Angew. Math. 184 (1942), 161-174. MR0008915 (5:74f)

11. S. Kaliman, F. Kutzschebauch, Density property for hypersurfaces $U V=P(\bar{X})$, Math. Z. 258 (2008), no. 1, 115-131. MR2350038 (2008k:32062)

12. S. Kaliman, F. Kutzschebauch, Criteria for the density property of complex manifolds, Invent. Math. 172 (2008), no. 1, 71-87. MR2385667 (2008k:32066)

13. S. Kaliman, F. Kutzschebauch, On the present state of the Andersén-Lempert theory, arXiv:1003.3434.

14. S. Kaliman, F. Kutzschebauch, Algebraic volume density property for affine algebraic manifolds, Invent. Math. 181 (2010), no. 1, 605-647. MR2660454

15. F. Kutzschebauch, H. P. Kraft, Equivariant affine line bundles and linearization, Math. Res. Lett. 3 (1996), no. 5, 619-627. MR1418576 (97h:14065)

16. L. Makar-Limanov, On groups of automorphisms of a class of surfaces, Israel J. Math. 69 (1990), no. 2, 250-256. MR1045377 (91b:14059)

17. L. Makar-Limanov, On the group of automorphisms of a surface $x^{n} y=P(z)$, Israel J. Math. 121 (2001), 113-123. MR1818396 (2001m:14086)

18. J. P. Rosay, W. Rudin, Holomorphic maps from $C^{n}$ to $C^{n}$, Trans. Amer. Math. Soc. 310 (1988), no. 1, 47-86. MR.929658 (89d:32058)

19. W. Van der Kulk, On polynomial rings in two variables, Nieuw Arch. Wiskunde (3) 1 (1953), 33-41. MR 0054574(14:941f)

20. D. Varolin, A general notion of shears, and applications, Michigan Math. J. 46 (1999), no. 3, 533-553. MR1721579 (2001a:32032) 
21. D. Varolin, The density property for complex manifolds and geometric structures, J. Geom. Anal. 11 (2001), no. 1, 135-160. MR1829353 (2002g:32026)

22. D. Varolin, The density property for complex manifolds and geometric structures. II, Internat. J. Math. 11 (2000), no. 6, 837-847. MR.1785520 (2002g:32027)

Institute of Mathematics, University of Bern, Sidlerstrasse 5, CH-3012 Bern, SWITZERLAND

E-mail address: Frank.Kutzschebauch@math.unibe.ch

Department of Mathematics, Mid Sweden University, SE-851 70 Sundsvall, Sweden

E-mail address: Andreas.Lind@miun.se 